\title{
Resilience Through Squamous Cell Carcinoma and Rhabdomyosarcoma of Oral Cavity: A Unique Case Report
}

\author{
Sidra Mumtaz Shaikh ${ }^{\mathrm{a}, \mathrm{d}}$, Sobia Faisal ${ }^{\mathrm{a}}$, Iqbal A. M. Khyani ${ }^{\mathrm{b}}$, \\ Bushra Sikandarc
}

\begin{abstract}
Rhabdomyosarcoma (RMS) is a soft tissue neoplasm of mesenchymal origin. It is a commonly encountered malignant tumor amongst pediatric patients, yet relatively rare in adults. It usually involves the head and neck region, genitourinary organs and retroperitoneal structures. In adults, the most commonly affected area is the head and neck region. We present here a case of a 30 -year-old male patient with a primary squamous cell carcinoma of the tongue (T1, N0, M1), successfully cured with surgery and chemoradiotherapy and later on development of metachronous ipsilateral lesion on the left lower alveolus. Biopsy was consistent with spindle cell RMS. Immunohistochemistry demonstrated positivity for desmin, vimentin and myogenin, thus confirming the mesenchymal origin. With the best of our literature search, this is an exceptional case presenting two malignant lesions with diverse genetic origins, diagnosed at stage 1 and giving a favorable outcome.
\end{abstract}

Keywords: Mesenchymal differentiation; Alveolar rhabdomyosarcoma; Squamous cell carcinoma; Chemotherapy; Immunohistochemistry

\section{Introduction}

Among the malignant oral cavity lesions, squamous cell carcinoma comprises the most common pathologic type, associated with significant morbidity, speech and swallowing disabilities. Despite recent diagnostic and therapeutic improvement, a $10 \%$ increase in mortality is observed in the last 5 years [1]. The increased mortality related to squamous cell carcinoma

Manuscript accepted for publication August 10, 2016

aDow University of Health Sciences, Former House Officers, Civil Hospital Karachi, Pakistan

bDepartment of ENT, Head and Neck Surgery, Dow University of Health Sciences, Karachi, Pakistan

${ }^{\mathrm{c}}$ Department of Pathology, Dow University of Health Sciences, Karachi, Pakistan

${ }^{\mathrm{d} C o r r e s p o n d i n g ~ A u t h o r: ~ S i d r a ~ M u m t a z ~ S h a i k h, ~ D o w ~ U n i v e r s i t y ~ o f ~ H e a l t h ~ S c i-~}$ ences, Former House Officers, Civil Hospital Karachi, Pakistan.

Email: sidramumtaz90@gmail.com

doi: http://dx.doi.org/10.14740/wjon978w of tongue (SCCOT) is perhaps due to the fact that most cases $(94.3 \%)$ present with locally invasive disease, with incidence rate highest amidst fourth and sixth decades of life [2].

Rhabdomyosarcoma (RMS) is a malignant soft tissue lesion occurring most frequently in children in the initial decades [3], with 250 new cases diagnosed in the United States per annum. However, it is a rare entity in adults and carries an adverse prognosis, possibly due to differences in histological subtypes, lack of treatment protocols and presentation in advanced stages [4].

This case elaborates long-term survival and management of a middle-aged man with a history of SCCOT. It was followed by a complete cure, with further development of spindle cell rhabdomyosarcoma (SC-RMS) of the oral cavity as a metachronous primary lesion. The depth of invasion, en block tumor resection and involvement of regional lymph nodes are the major dynamics to evaluate for prognosis and recurrence rate. Our case is unique because of its peculiar age of presentation of 30 years, along with its favorable survival of 8 years.

\section{Case Report}

A 30-year-old male, married, shop keeper by profession, smoker for the past 10 years with no known comorbids, presented at a tertiary care hospital in 2009 with a 2-month history of a painless ulcer along the left lateral border of the tongue. He was initially treated with antibiotics by his general practitioner which proved to be ineffective. Punch biopsy was performed under local anesthesia and the histopathological findings were consistent with grade 1 well-differentiated keratinizing SCCOT (Fig. 1). He underwent left-sided hemi-glossectomy with neck dissection. Three cycles of chemotherapy were given pre- and postoperatively. Furthermore, he underwent 35 cycles of radiotherapy.

Patient was quite well and disease free for 4 years until 2013, when he noticed a pea-sized tender swelling on inner aspect of the left lower alveolus. The lesion was excised and biopsy showed polyploid pyogenic granuloma with no signs of malignancy. The swelling recurred within 4 months. Patient complained of progressive and painful enlargement of the lesion, loosening of teeth and decreased mouth opening. During intra-oral examination, three exophytic growths with smooth erythematous surface were found on left mandibular quadrant with the largest measuring about $3 \times 2 \mathrm{~cm}$, attached firmly on the alveolar bone with loss of canine and first and second premolars (Fig. 2). The smaller lesions measuring $1.5 \times 1.5$ and 1 


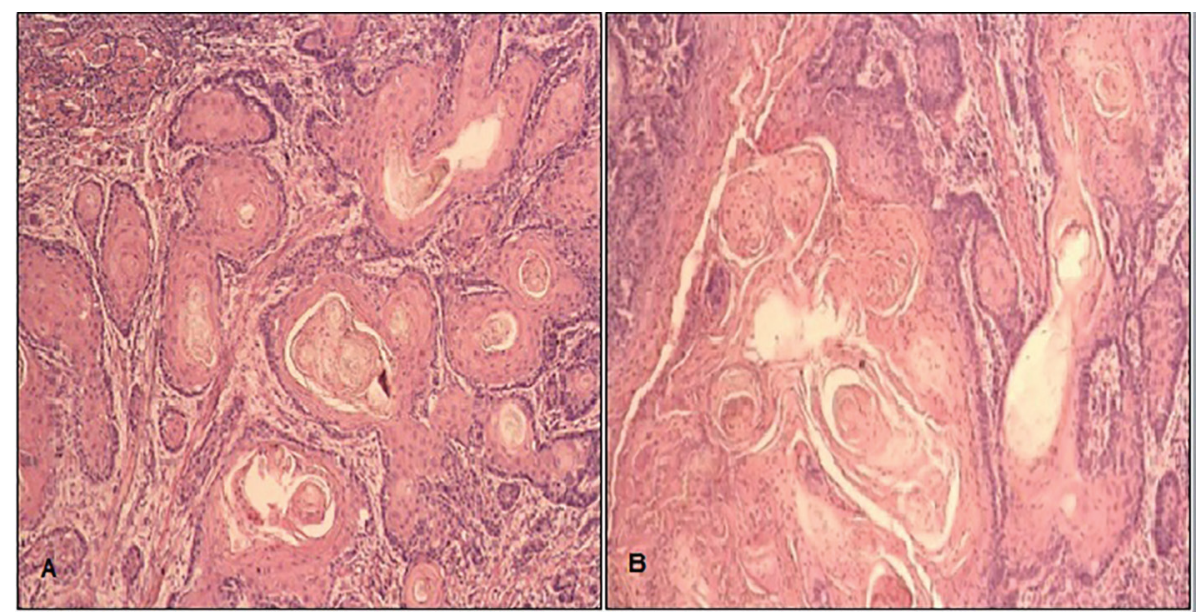

Figure 1. Squamous cell carcinoma of tongue (SCCOT): H\&E at 400 magnification showing (A) nest and cords of atypical squamous cells exhibiting pleomorphic vesicular nuclei with prominent nucleoli having abundant cytoplasm and keratin pearls and (B) individual cell keratinization.

$\times 1 \mathrm{~cm}$ were found on medial and posterior aspects, respectively. No extra-oral abnormality was observed and cervical lymph nodes were non-palpable. Incisional biopsy revealed spindle cell carcinoma. Complete surgical excision was planned. After all aseptic measures, patient lied in a supine position, intubated and anesthetized. Left-sided segmental mandibulectomy was performed through visor's incision (transcervical approach). En block tumor resection was undertaken. Incision was extended caudally to approach level I, II and III lymph nodes. Bilateral supra-omohyoid neck dissection was performed. Titanium reconstruction plate was applied over the defect, followed by repair of the floor of the mouth. Neck incision was closed and drains were placed.

Postoperatively, patient recovered uneventfully. Histopathological assessment showed sheets of atypical spindle

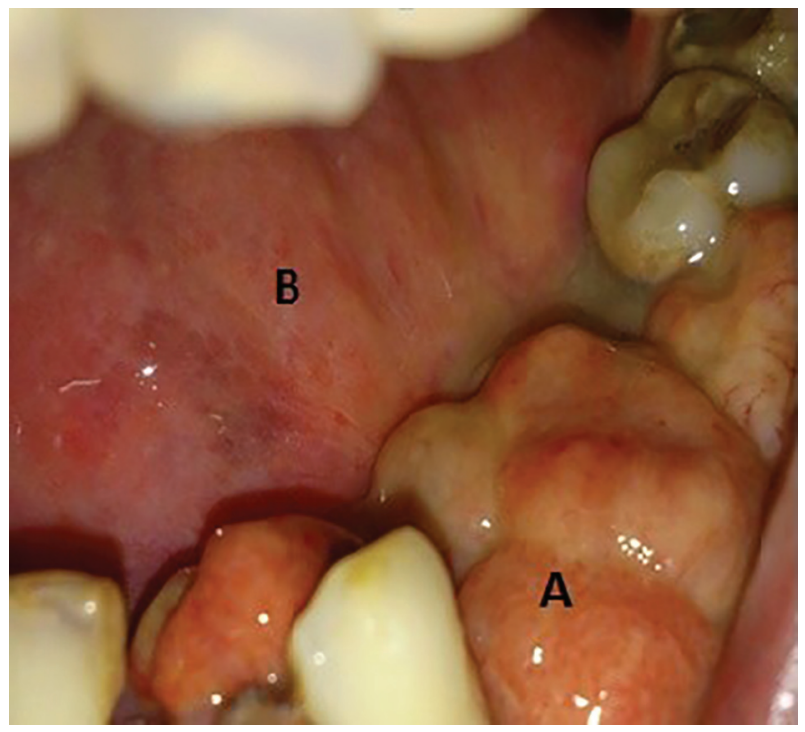

Figure 2. (A) $A 3 \times 2 \mathrm{~cm}$ growth at left mandibular quadrant with loss of canine, first and second premolars. (B) Site of previous hemi-glossectomy. cells with large hyperchromatic nuclei, prominent nucleoli and abundant eosinophilic cytoplasm (Fig. 3). Positive immunohistochemical features (Table 1, Figs. 4, 5) included vimentin, anti-smooth muscle antibody (ASMA), myogenin and desmin in tumor cells, consistent with diagnosis of SC-RMS.

Follow-up CT scan at third month showed no evidence of focal mass or cervical lymphadenopathy. He was referred to oncology department where he underwent chemotherapy. The combination of vincristine, actinomycin D and cyclophosphamide was given. After three cycles, he developed chemotherapy-induced myelosuppression. Multiple transfusions were performed. Five cycles of the same regimen were repeated after restoration of hemodynamic status.

After completion of chemoradiotherapy, he reported back to us 3 months later with exposed reconstruction plate that had stripped off along the margins of the flap (Fig. 6). The case was discussed with multidisciplinary teams for mandibular reconstruction using autologous cortical bone; however, the patient denied any further surgical procedure and requested for plate removal, which was then removed under general anesthesia.

Table 1. Immunocytological Findings of the Resected Specimen

\begin{tabular}{ll}
\hline Immunological markers & Findings \\
\hline Panck & Negative \\
Cytokeratin AE1/AE3 & Negative \\
Cytokeratin 8/18 & Negative \\
P63 & Negative \\
Vimentin & Positive \\
ASMA & Positive \\
Myogenin & Positive \\
CD34 & Negative \\
S-100 & Negative \\
Desmin & Positive \\
\hline
\end{tabular}



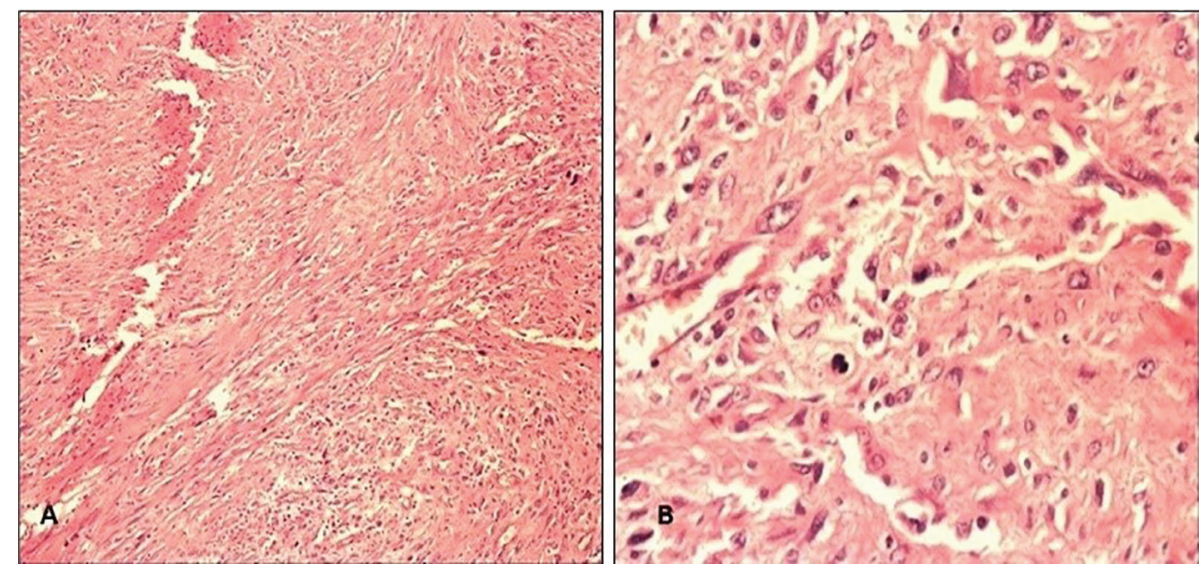

Figure 3. (A) H\&E at 100 magnifications shows sheets of neoplastic spindle cells. (B) H\&E at 400 magnification shows clusters of atypical cells with hyperchromatic nuclei, abundant eosinophilic cytoplasm and increased nucleus to cytoplasmic ratio.

He later on developed fistula at the site of mandibular resection with sero-sanguinous discharge for which he was prescribed appropriate antibiotics after specimen culture. Thereafter, we lost contact of the patient.

\section{Discussion}

Neoplastic lesions of the oral cavity are one of the top $10 \mathrm{ma}-$ lignancies in the world, and second most common in Pakistan [5]. Squamous cell carcinoma is the most common histological type with approximately 48,300 new cases expected in 2016 [6]. Risk factors include alcohol, tobacco and betel nuts that play a significant role towards the development of premalignant lesion like sub mucosal fibrosis and leukoplakia [7]. The development of SCCOT in our patient is implicated with his smoking history (10 pack-years).

Tumor size and depth of invasion, spread to regional lymph nodes, and negative tumor margin assessment contribute towards the prognosis and functional staging [8]. Cervical lymphadenopathy and extracapsular invasion carry poor prognosis in SCCOT [9].

The results of the study performed by Jerjes et al entailed nodal involvement in eight out of 10 deaths due to local spread of disease and in eight out of 11 deaths due to metastasis [10]. While a discrepancy exists in determining prognosis and survival rate with age of initial presentation [11], Jerjes et al concurred that younger patients tend to have a worse prognosis, while older patients are likely to die from other comorbid con- ditions especially cardiorespiratory failure [10]. The 5-year survival rate is estimated to be $45-50 \%$ [5]. In this peculiar case, our patient presented at the age of 30 years, with grade I, well-differentiated SCCOT (T1N1M0). He responded well to the combined approach, i.e., surgery and chemoradiotherapy. No recurrence was observed in the subsequent 4 years, until the diagnosis of RMS was made.

RMS arises from the skeletal muscle precursor cells, derived from connective tissue mesenchyme. The tumor is third in frequency to Wilm's and neuroblastoma for the pediatric neoplastic lesion. It affects head and neck, genital tract (sarcoma botryoid) retroperitoneal structures and appendicular skeleton in decreasing order of frequency. Oral cavity lesions account for $10-12 \%$ cases of RMS in head and neck region [12]. Its occurrence is a rare entity in adults with head and neck as the most frequent site involved [13]. Atypical mesenchymal proliferation during embryonic tissue development is believed to form histological aspects of RMS. On the basis of cellular growth pattern, RMS is divided into alveolar (ARMS), embryonal (ERMS), botryoid and pleomorphic types (PRMS) [12].

Agaram et al excluded SC-RMS from ERMS subtypes and categorized them as distinct pathologies due to genetic alterations in MYOD1 genes [14]. The histological pattern of SC-RMS included proliferating spindle cells with long fascicles having eosinophilic cytoplasm and interspersed rhabdomyoblasts $[14,15]$. The etiological factors associated with SCRMS are not yet known. Genetic defects are found to play a role in tumor development. Szuhai et al established MYOD1 mutations as the cause of adult SC-RMS. p.L122R mutations

Table 2. Stages of Rhabdomyosarcoma According to International Rhabdomyosarcoma Study Group (IRSG) [3]

\begin{tabular}{llll}
\hline Stage & Location & Nodal status & Metastasis \\
\hline Stage I & Orbit, head and neck, non-parameningeal, and genitourinary & N0 or N1 & No metastasis \\
& Any size & & No metastasis \\
Stage II & Any other site $<5 \mathrm{~cm}$ & N1 & No metastasis \\
Stage III & Any other site $<5 \mathrm{~cm}$ & N0 or N1 & M1 \\
& Any other site $>5 \mathrm{~cm}$ & N0 or N1 & \\
Stage IV & Tumor at any location and of any size & & No \\
\hline
\end{tabular}




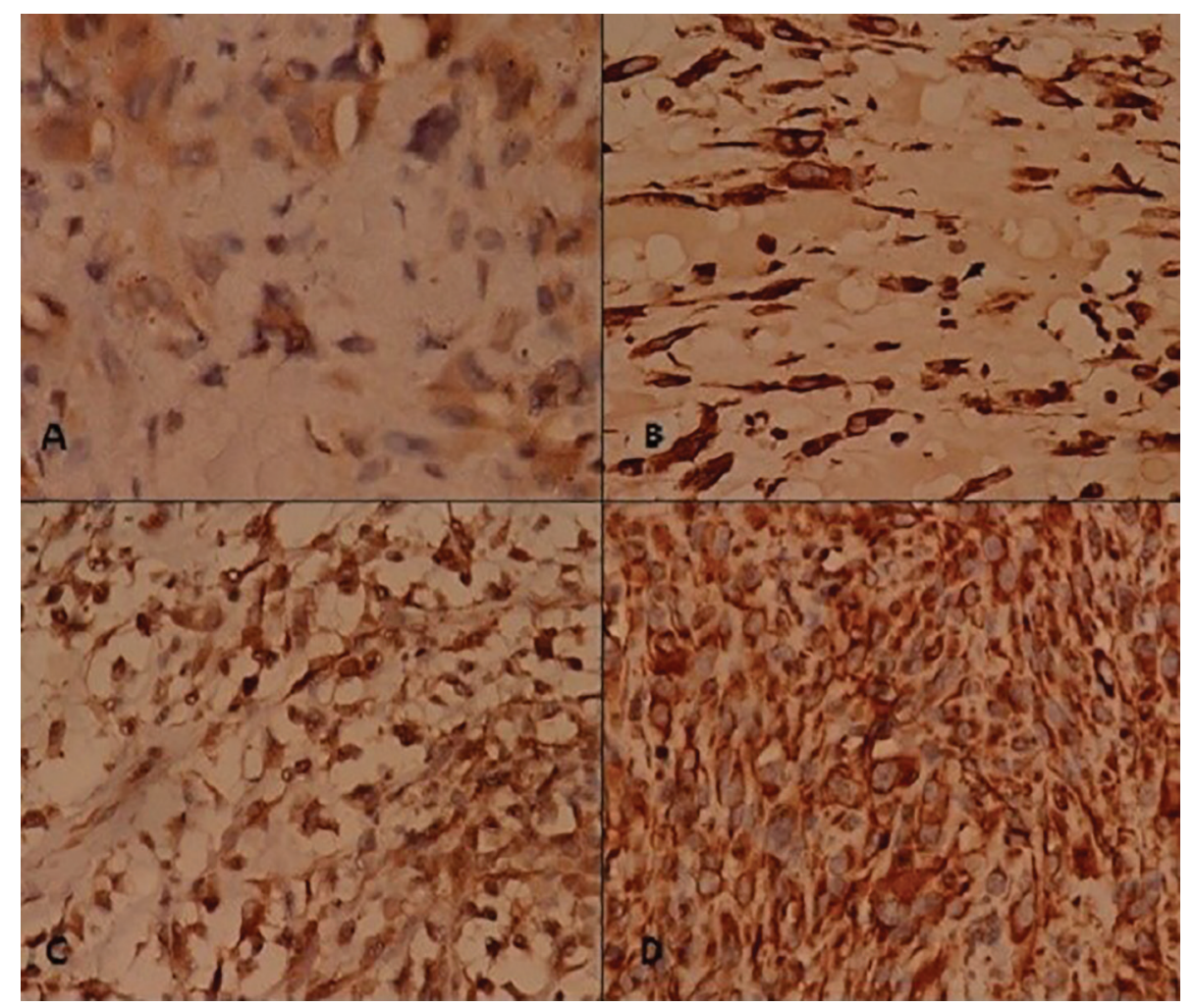

Figure 4. Immunohistochemistry (1). Tumor cells stain positive for (A) desmin, (B) myogenin, (C) ASMA, and (D) vimentin.

in the DNA binding domain of the MYOD1 gene initiate possible amplification of nuclear regulatory protein MYC. Both homozygous and heterozygous mutations can cause SC-RMS. MYOD1 gene regulates myocyte differentiation by transcribing nuclear protein complex and its mutation can lead to failure in cell cycle arrest which is a crucial stage in myogenic differentiation [16].

Conventionally, most head and neck squamous cell carcinomas (HNSCCs) are associated with TP53, NOTCH-1 and CKDN2A mutations. Yet in one of the studies performed by Vettore et al, DST and RNF213 mutations were more significant among Asian population, whereas NOTCH-1, CKDN2A and p53 alterations were uncommonly found [17].

Hence, no possible genetic correlation exists between oral tongue squamous cell carcinoma (OTSCC) and SC-RMS. The development of SC-RMS should be considered a distinctive pathologic lesion rather than the sequel of OTSCC. In our case, the external beam radiotherapy given for SCCOT probably acted as a "second hit" to a pre-existing dormant mutation. Due to financial constraints, genetic typing could not be performed to further confirm the hypothesis.

Biopsy and immunohistochemistry constitute a great part in making a diagnosis. RMS is recognized on the basis of positivity for several immune markers including muscle specific

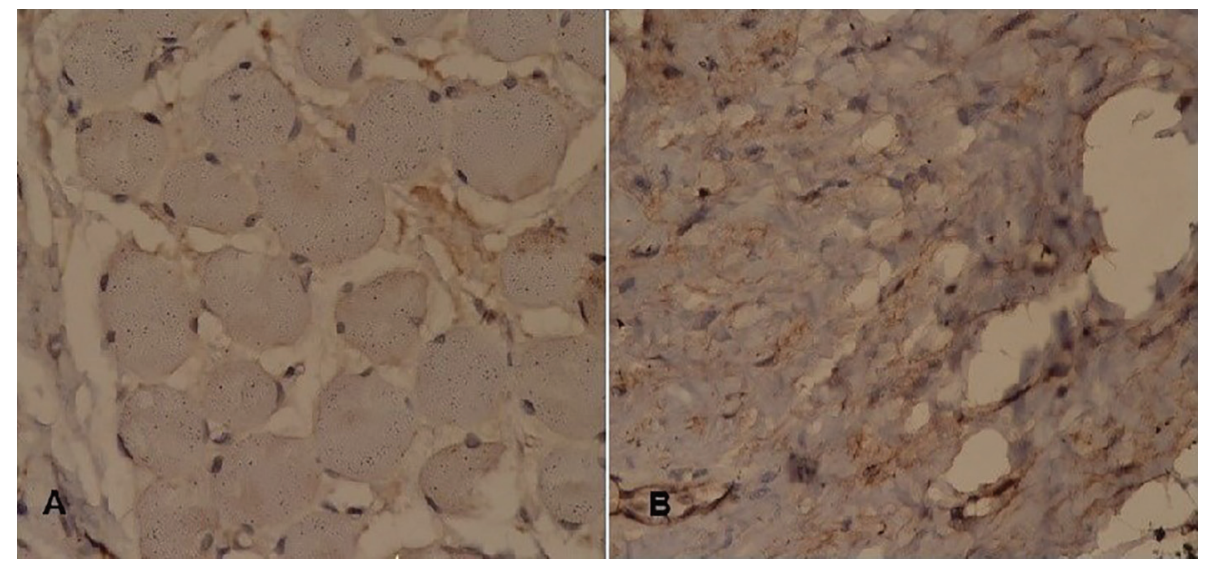

Figure 5. Immunohistochemistry (2). Tumor cells stain negative for (A) CK AE1/CK AE3 and (B) S-100. 


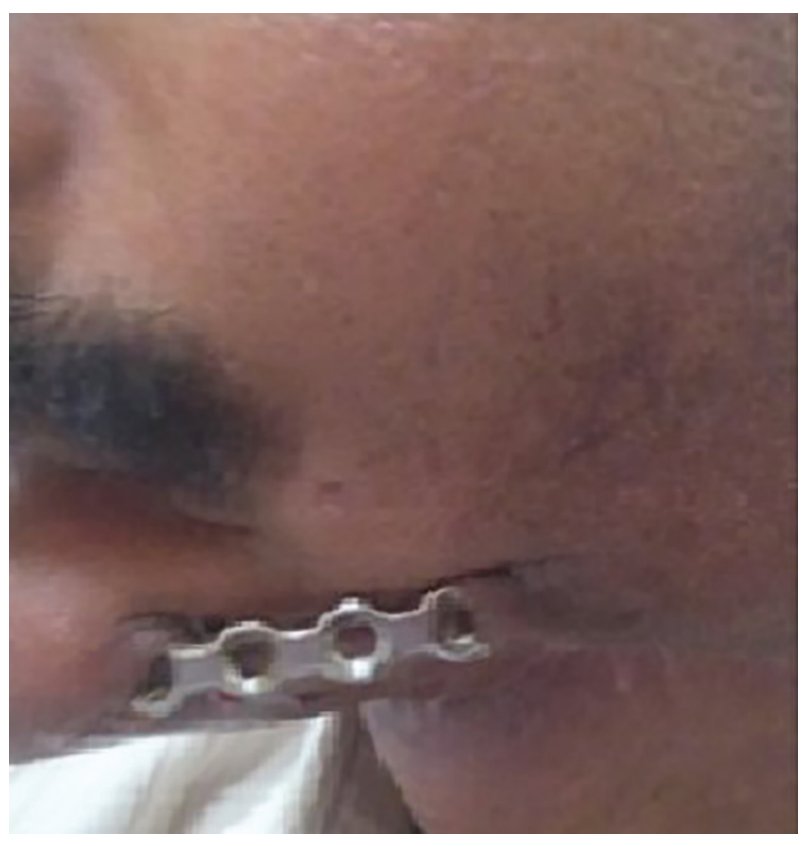

Figure 6. Exposed reconstruction plate.

actin, cytokeratin, myogenin, desmin, S-100 protein, vimentin and cytokeratin [15]. The histopathology in our case exhibited uniform positivity for ASMA, myogenin, desmin and vimentin that confirms cellular pattern of mesenchymal origin. S-100 was negative (thus differentiated from peripheral nerve sheath tumor) as well as cytokeratin AE1/AE3.

Table 2 elaborates various stages of RMS on the basis of anatomical site of involvement, nodal status and metastasis according to International Rhabdomyosarcoma Study Group (IRSG) protocol [3].

The treatment options related with disease outcome are less eminent for adult SC-RMS and the childhood management protocol is followed including gross resection with combined system chemotherapy and radiotherapy (except for ERMS). Outcome in children showed $70-80 \%$ remission, yet the survival rate for adult may vary between $35 \%$ and $45 \%$ [18].

In our patient, we performed segmental mandibulectomy for the tumor resection and approached level I, II and III lymph nodes via bilateral supra omohyoid dissection. This had facilitated us to acquire tumor free margins and also minimized morbidity associated with surgery. Postoperatively, the patient initially responded well to chemotherapy. There is no superior advantage of concomitant radiotherapy in group 1 patients having ERMS subtype [3].

The surviving group of patients with head and neck cancers can develop radiation-induced sarcoma. To establish a relation between the irradiation and malignancy, the lesion must arise in the irradiated area and should be different from the primary lesion being treated [19], with an average gap of around 3 - 4 years between the radiation therapy and the sarcoma development [20]. Thus, we propose that the SC-RMS presented in our case could have been caused by radiotherapy given for SCCOT, as the lesion arose in the radiation affected area, had a different histological subtype than the primary lesion, and presented after 4 years (metachronous).

Reconstruction techniques include use of biocompatible alloplastic plates, vascularized and non-vascularized bone grafts. However, in majority of cases, plate exposure occurs, which increases the risk for infections and subsequent fistula formation between skin and oral cavity [21]. In our case report, patient experienced a similar outcome with fistula formation. Hence, combined technique is suggested in which free flaps comprising of the pectoralis major or sternocleidomastoid muscle are wrapped around the end of the plate to ensure better approximation and to decrease the rates of plate exposure [22].

Unlike the pediatric counterpart, adult SC-RMS has an aggressive course and is associated with an adverse prognosis with a 5 -year survival rate of $47 \%$ compared to $82 \%$ in children [4]. Little et al assessed outcome following multimodality treatment in adult patients with RMS and established tumor size as an effective index of prognosis. A disease free survival rate of $57 \%$ was observed in patients having a tumor size of $\leq$ $5 \mathrm{~cm}$ compared to $26 \%$ for those with a size of $>5 \mathrm{~cm}$ [18]. En block tumor resection of localized lesion in stage I patients ensured a good 5-year disease free survival, while the contrary existed for stage IV. Stage II and III patients showed intermediary responses and hence it is essential to treat these patients with appropriate combination therapy [3].

Prior case reports related to mandibular RMS were reported in India [23, 24] which call for attention to the increased incidence of mandibular RMS in the South Asian population. However, these cases depicted primary malignant RMSs, whereas the presented case showed SC-RMS occurring as a metachronous neoplasm 4 years after the presentation of SCCOT. Additionally, the patient has had a prolonged survival of 8 years since the presentation of the initial lesion, which is an exceptionally rare situation.

\section{Conclusion}

The diagnosis of SC-RMS is challenging and increased caution is required amongst clinicians to diagnose lesions earlier on, therefore non-healing ulcers and growths should be sent for histopathological assessment. Immunohistochemistry plays a critical role in specifying various histologic subtypes. The evolution of SC-RMS as a distinct metachronous lesion raises concern for its possible association with prior radiotherapy and likewise dose should be appropriately adjusted and minimized. This case highlights the fact that early detection and surgical resection at initial stages of RMS in an adult can give promising results.

\section{Competing Interest}

The authors declare that they have no competing interest.

\section{Consent}

Written consent had been taken from the patient for publica- 
tion of case report and images.

\section{Author Contributions}

SMS conceived the idea, wrote the case summary and discussion. SF contributed in discussion and manuscript drafting. IK performed the surgeries and critically reviewed the manuscript. BS contributed in histopathological assessment. All authors have reviewed the manuscript.

\section{Abbreviations}

RMS: rhabdomyosarcoma; SCCOT: squamous cell carcinoma of tongue; SC-RMS: spindle cell rhabdomyosarcoma; ARMS: alveolar rhabdomyosarcoma; ERMS: embryonal rhabdomyosarcoma; PRMS: pleomorphic rhabdomyosarcoma; ASMA: anti smooth muscle antibody; HNSCC: head and neck squamous cell carcinoma

\section{References}

1. Yu X, Li Z. MicroRNA expression and its implications for diagnosis and therapy of tongue squamous cell carcinoma. J Cell Mol Med. 2016;20(1):10-16.

2. Narang S, Kanungo N, Jain R. Squamous cell carcinoma: morphological \& topographical spectrum: a two year analysis. Indian J Surg. 2014;76(2):104-110.

3. Raney RB, Maurer HM, Anderson JR, Andrassy RJ, Donaldson SS, Qualman SJ, Wharam MD, et al. The Intergroup Rhabdomyosarcoma Study Group (IRSG): Major Lessons From the IRS-I Through IRS-IV Studies as Background for the Current IRS-V Treatment Protocols. Sarcoma. 2001;5(1):9-15.

4. Egas-Bejar D, Huh WW. Rhabdomyosarcoma in adolescent and young adult patients: current perspectives. Adolesc Health Med Ther. 2014;5:115-125.

5. Syed SA, Bukhari MH, Warraich RA, Quadir F, Iqbal A, Syed AF. Oral squamous cell carcinoma; clinico-pathological parameters and agnor status in grading. Professional Med J. 2015;22(1):18-26.

6. Siegel RL, Miller KD, Jemal A. Cancer statistics, 2016. CA Cancer J Clin. 2016;66(1):7-30.

7. Baig S, Rubab Z, Arif MM, Lucky MH. Chewable risk factors - Threatened oral cancer HPV's Looming Epidemic in Pakistan. European Journal of Biotechnology and Bioscience. 2015;3(1):39-45.

8. Hema K, Rao K, Devi HU, Priya N, Smitha T, Sheethal H. Immunohistochemical study of CD44s expression in oral squamous cell carcinoma-its correlation with prognostic parameters. J Oral Maxillofac Pathol. 2014;18(2):162168.

9. Sano D, Myers JN. Metastasis of squamous cell carcinoma of the oral tongue. Cancer Metastasis Rev. 2007;26(34):645-662.
10. Jerjes W, Upile T, Petrie A, Riskalla A, Hamdoon Z, Vourvachis M, Karavidas K, et al. Clinicopathological parameters, recurrence, locoregional and distant metastasis in $115 \mathrm{~T} 1-\mathrm{T} 2$ oral squamous cell carcinoma patients. Head Neck Oncol. 2010;2:9.

11. Majchrzak E, Szybiak B, Wegner A, Pienkowski P, Pazdrowski J, Luczewski L, Sowka M, et al. Oral cavity and oropharyngeal squamous cell carcinoma in young adults: a review of the literature. Radiol Oncol. 2014;48(1):110.

12. Miloglu O, Altas SS, Buyukkurt MC, Erdemci B, Altun O. Rhabdomyosarcoma of the oral cavity: a case report. Eur J Dent. 2011;5(3):340-343.

13. Hartmann S, Lessner G, Mentzel T, Kubler AC, MullerRichter UD. An adult spindle cell rhabdomyosarcoma in the head and neck region with long-term survival: a case report. J Med Case Rep. 2014;8:208.

14. Agaram NP, Chen CL, Zhang L, LaQuaglia MP, Wexler L, Antonescu CR. Recurrent MYOD1 mutations in pediatric and adult sclerosing and spindle cell rhabdomyosarcomas: evidence for a common pathogenesis. Genes Chromosomes Cancer. 2014;53(9):779-787.

15. Wu TT, Wang QY, Zhou SH, Zheng ZJ. Spindle cell rhabdomyosarcoma in the hypopharynx of an adult. Int J Clin Exp Pathol. 2014;7(8):5254-5258.

16. Szuhai K, de Jong D, Leung WY, Fletcher CD, Hogendoorn PC. Transactivating mutation of the MYOD1 gene is a frequent event in adult spindle cell rhabdomyosarcoma. J Pathol. 2014;232(3):300-307.

17. Vettore AL, Ramnarayanan K, Poore G, Lim K, Ong CK, Huang KK, Leong HS, et al. Mutational landscapes of tongue carcinoma reveal recurrent mutations in genes of therapeutic and prognostic relevance. Genome Med. 2015;7:98.

18. Little DJ, Ballo MT, Zagars GK, Pisters PW, Patel SR, El-Naggar AK, Garden AS, et al. Adult rhabdomyosarcoma: outcome following multimodality treatment. Cancer. 2002;95(2):377-388.

19. Thiagarajan A, Gopalakrishna iyer N. Radiation induced sarcoma of the head and neck. World J Clin Oncol. 2014;5(5):973-981.

20. Huber GF, Matthews TW, Dort JC. Radiation-induced soft tissue sarcomas of the head and neck. J Otolaryngol. 2007;36(2):93-97.

21. Chim H, Salgado CJ, Mardini S, Chen HC. Reconstruction of mandibular defects. Semin Plast Surg. 2010;24(2):188197.

22. Torroni A, Marianetti TM, Romandini M, Gasparini G, Cervelli D, Pelo S. Mandibular reconstruction with different techniques. J Craniofac Surg. 2015;26(3):885-890.

23. Patil G, Halawar S, Sagari S, Babannavar R, Purohit S. Embryonal rhabdomyosarcoma occurring on mandibular gingiva in an adult. J Clin Diagn Res. 2013;7(9):20882089.

24. Arya K, Vij H, Vij R, Rao NN. Rhabdomyosarcoma of mandible: A diagnostic predicament. J Oral Maxillofac Pathol. 2011;15(3):320-325. 